\title{
Instrumentalizing Cognitive Dissonance Emotions
}

\author{
Marie-Claude Bonniot-Cabanac ${ }^{1}$, Michel Cabanac ${ }^{1}$, José F. Fontanari ${ }^{2}$, \\ Leonid I. Perlovsky ${ }^{3}$ \\ ${ }^{1}$ Department of Psychiatry \& Neurosciences, Faculty of Medicine, Laval University, Quebec, Canada \\ ${ }^{2}$ Instituto de F'isica de São Carlos, Universidade de São Paulo, São Carlos SP, Brazil \\ ${ }^{3}$ Athinoula A. Martinos Center for Biomedical Imaging, Harvard University, Charlestown, USA \\ Email: lperl@rcn.com
}

Received September $22^{\text {nd }}, 2012$; revised October $18^{\text {th }}, 2012$; accepted November $16^{\text {th }}, 2012$

\begin{abstract}
Many psychologists think that there are few basic emotions, and most emotions are combinations of these few. Here we advance a hypothesis that the number of principally different emotions is near infinite. We consider emotions as mental states with hedonic content, indicating satisfaction and dissatisfaction. Basic emotions correspond to bodily signals, and there are relatively few of them. Our hypothesis is that a large number of emotions are related to the knowledge instinct (KI, or a need for knowledge). KI drives the mind to fit mental representations to cognitive experiences and to resolve mental contradictions. Discomfort due to holding contradictory knowledge elements are known as cognitive dissonances. We emphasize that cognitive dissonances involve specific emotions. The number of cognitive dissonances is combinatorial in terms of elements of knowledge. Correspondingly, the number of these knowledge-related emotions is very large. We report experimental results on measuring these emotions and indicating that emotions of cognitive dissonance exist. We also make a step toward proving that these emotions are different from basic emotions in principle, and outline future research directions toward proving that their number is large.
\end{abstract}

Keywords: Cognitive Dissonance Emotions; Basic Emotions; Instrumentalizing; Basic Emotions

\section{Introduction}

Emotions are topics of much discussion in psychological literature, their mechanisms and functions are subjects of controversy (Ekman, 1999; Juslin \& Västfjäll, 2008). Psychological functions of emotions have been related to social functioning and associated with facial expressions (Ekman, 1957), emotions have been related to survival (Plutchik, 1962), and to mental states (Ortony et al., 1987). Belief-desire theory of emotions has been developed in (Gratch et al., 2009; Reisenzein, 2009). Emotions have been argued to perform appraisals of concepts and events (Bechara et al., 2004; Damasio et al., 1994; Scherer et al., 2001), although the question of phylogenic emergence of emotions vs. mental representations of events has not been adequately addressed in literature (Cabanac et al., 2009). Emotions have been associated with creativity (Perlovsky, 2010g; Festinger, 1957). We consider emotions as mental states with hedonic content (Cabanac, 2002) that indicates satisfaction and dissatisfaction of instinctual drives (Grossberg \& Levine, 1987). Usually psychologists study basic emotions (Ekman, 1999); there are several proposals as to the nature of basic emotions, and their number varies among authors from two to a dozen (Plutchik, 1980; Tooby \& Cosmides, 1990; Lazarus, 1991, Johnson-Laird \& Oatley, 1992; Ekman, 1999; Ortony \& Turner, 1990; Izard, 1992). We consider basic emotions as related to bodily instincts and named by specific words. There are few different basic emotions, much fewer than emotional words (Petrov et al., 2012). Most psychologists consider other emotions as combinations of the basic emotions (Plutchik, 1962; Ekman, 1999; Ortony \& Turner, 1990; Izard, 1992).

We would emphasize that basic emotions unify human and animal worlds. But what is the origin of the wealth of specifically human emotional experience, such as emotions of the beautiful and sublime, musical emotions, and emotions we perceive in songs and language prosody (Perlovsky, 2000, 2001, 2010a, 2010b, 2011c, 2011e, 2011g)? We disagree that they are just mixtures of few basic emotions. We suggest that the number of fundamentally different human emotions is much larger than the number of emotional words.

Most of human emotions are related to knowledge; they are called aesthetic emotions since (Kant, 1790). We suggest that they are related to satisfaction or dissatisfaction of the knowledge instinct (KI) (Perlovsky, 1997, 2001, 2006a, 2006b, 2007a, 2007b, 2007c, 2008, 2010c, 2010d, 2010e, Perlovsky, Bonniot-Cabanac et al., 2012; Levine \& Perlovsky, 2008, 2010), and are inseparable from learning and cognition (Perlovsky \& Ilin, 2012a, 2012b; Tikhanoff et al., 2006; Kovalerchuk, 2012; Vityaev et al., 2011). Existence of these emotions have experimentally been demonstrated in (Bonniot-Cabanac \& Cabanac, 2009; Bonniot-Cabanac et al., 2012, Perlovsky \& Ilin, 2010). They are related to the arts, the beautiful and sublime (Perlovsky, 2000, 2001, 2002a, 2002b, 2010a, 2010f, 2010g, 2011a, 2011b, 2011f, 2012a, 2012b, 2012c, 2012d, 2012e, 2012i, 2012j, 2012k).

Here we concentrate on aesthetic emotions related to cognitive dissonances (CD) (Festinger, 1957). In the CD theory a discomfort due to contradictions in knowledge has been considered as a non-differentiated state. Emotions related to $\mathrm{CD}$ have not been studied. Here we emphasize that a contradiction between any two elements of knowledge is a separate mental state with its own $\mathrm{CD}$ emotion. This is a reason for a large number of $\mathrm{CD}$ and the corresponding emotions. Every combi- 
nation of two or more elements of knowledge contains some degree of contra- diction among these elements (Perlovsky, 2004, 2009a, 2009b, 2009c, 2010a, 2012b, 2012d, 2012e, 2012f, 2012g, 2012h, 2012i).

It is discussed in these references that cognitive dissonances are uniquely human phenomena. Evolution of knowledge abilities in animals was slow, and no contradiction in the system of knowledge evolved. In animals' mind, conceptual understanding of a situation, its emotional evaluation, and appropriate behavior are a single unified psychic state. In the human mind, with evolution of language, differentiation of human knowledge accelerated (Fontanari et al., 2007, 2008a, 2008b, 2009; Tikhanov et al., 2006). This differentiation lead to cognitive dissonances, a loss of the unity of self, and potential lost of concentration of will. Those of our ancestors, who could differentiate the knowledge about the world while maintaining concentration of will, received evolutionary advantage. Therefore emotions unifying contradictions in knowledge had to evolve along with language. As language and culture were evolving into a powerful system with tremendous differentiation of knowledge about the world and self, the number of contradictions grew combinatorially (Perlovsky, 2004, 2006c, 2010a, 2012e). Every combination of conceptual pieces of knowledge led to its own shades of contradictions. Therefore, maintaining motivation for this diversified knowledge required virtually infinite number of shades of motivations. Knowledge elements acquired from surrounding language contradict to bodily instincts and to each other. Continuing evolution of language and knowledge required resolution of these contradictions. The resolution of CD required emotions, potentially a unique emotion for each dissonance (Perlovsky, Cabanac et al., 2012; Perlovsky \& Levine, 2012). Existence and nature of these emotions is explored in this paper, both experimentally and theoretically.

Aesthetic emotions related to cognitive dissonances can be illustrated in the following examples. Consider an emotion related to a mental effort of making a choice if one receives offers of positions at Harvard and Stanford. Each offer would be considered great by many people, and would result in highly positive emotions; these emotions would be mostly basic emotions related to satisfaction of basic needs and aspirations. But making the choice between the two offers could be difficult and evoke negative emotions. For many people it could be an excruciatingly painful decision. Making important life choices, concerning place of living or job, even between wonderful alternatives, could be difficult. Thus basic emotions associated with each alternative have nothing to do with mental effort of choice. This proves the main hypothesis of this paper: Emotions of CD are different from basic emotions. A first step toward exploring this possibility is a hypothesis that the number of $\mathrm{CD}$ emotions is much larger than the number of basic emotions.

However, there are no methods of measuring CD emotions. Whereas basic emotions are named with specific words, there are no words for $\mathrm{CD}$ emotions. Therefore the main goal of this paper is to instrumentalize CD emotions, in other words to develop experimental methods for their exploration. The following parts of the paper investigate properties of aesthetic emotional spaces. Even minute decisions, seemingly effortless, still require some emotional effort. To make a choice, any choice, one has to have a motivation, therefore, an emotion. Even most simple everyday choices between "tea or coffee", "red or white wine", create minute cognitive dissonances, and related emotions. These kinds of emotions are considered below. The primary goal of the following parts of this paper is to explore experimental methods "instrumentalizing" aesthetic emotions of cognitive dissonances, demonstrating that these emotions exist, can be experimentally measured, and to initiate scientific research of their properties.

\section{Experimental Method}

This study followed a classical psychologic method that explored mental experience in interviews where participants answered printed questionnaires (Bonniot-Cabanac \& Cabanac, 2009, 2010; Balasko \& Cabanac, 1998; Cabanac et al., 1997, 2002; Cabanac \& Bonniot-Cabanac, 2007; Ramirez et al., 2005, 2009; Perlovsky, Bonniot-Cabanac et al., 2012). Thirty four anonymous participants (who were referred to by numbers only), 17 men (age 12 - 70) and 17 women (age 16 - 72) were presented two questionnaires each containing ten items. Both questionnaires presented the same items, but the participant was asked to rate hedonicity from one and intensity from the other. All items described a decision to be made among two conflicting motivations and the participant was to rate analogically the magnitude of her/his experience.

Hedonicity was explored by the first questionnaire-Questionnaire $\mathrm{H}$ : a horizontal line was present below the item with a zero on its middle, a minus (-) sign at the left end and a plus $(+)$ sign at the right end. The participant was to pencil a small vertical mark on the right side of that line if the feeling was pleasant, or on the left side if unpleasant. The experienced hedonic feeling would be indicated by the distance from the middle (zero mark) of the line.

The second questionnaire-Questionnaire E-explored emotion: as before, a horizontal line was present below the item but with the zero mark on its left end. The participant was to pencil a small vertical mark at that line rating the intensity of the experienced feeling. The distance from the zero mark would indicate the magnitude of the experience. After rating the magnitude of the emotion the participant wrote one word describing the nature of the experienced emotion, e.g., curiosity, surprise, joy, indifference, anger, etc. 33 of the 34 participants responded with the emotion word.

The hedonic and magnitude feelings were measured quantitatively in millimeters as well as recorded semantically. Questionnaires $\mathrm{E}$ and $\mathrm{H}$ were presented separately over time varied from about one hour to half a day, depending on the availability of the participant. This was done in order to minimize a possible influence of answering one questionnaire on the response to the other questionnaire. The first questionnaire was presented in the morning period while keeping the gender of the participants balanced, and the second questionnaire was presented in the afternoon.

Decisions to be made were described by ten items covering a broad range of motivations, from minor decisions in the daily life (e.g., how about movie or theatre for tonight?) to clear but non-vital problems (e.g., would you go for a high-gain but risky investment or for a low-gain but secure one?) and finally to vital problems (e.g., would you go for radical surgery or for life-long therapy to treat a severe illness?). This ten items are presented in the appendix).

\section{Analysis of the Data}

The degree of arousal $\mathrm{E}$ takes on positive values only 
whereas the degree of pleasure $\mathrm{H}$ can take on positive as well as negative values, $\mathrm{E} \in[0,1]$ and $\mathrm{H} \in[-1,1]$.

Characterizing emotions by two-dimensional space of arousal and pleasure corresponds to model (Russell, 1980, 1989). The data are presented in Figure 1 where the symbols indicate the values of the arousal $\mathrm{E}$ and pleasure $\mathrm{H}$ for the 340 points corresponding to the 10 choice questions of the 34 subjects.

A different characterization of the data in Figures 2 and 3 shows distributions $\sum_{\mathrm{E}} \Xi_{\mathrm{E}}$ and $\sum_{\mathrm{H}} \Xi_{\mathrm{H}}$ of the arousal and pleasure degrees. The normalization is such that $\sum_{\mathrm{E}} \Xi_{\mathrm{E}}=\sum_{\mathrm{H}} \Xi_{\mathrm{H}}=340$. One can see that the subjects' answers are well balanced. The mean values of these distributions yield $<\mathrm{E}>=0.417$ and $\langle\mathrm{H}>=0.058$. The mean value $<\mathrm{E}>$ can be used to separate the regime high arousal $(\mathrm{E}><\mathrm{E}>$ ) from low arousal $(\mathrm{E}<<\mathrm{E}>)$. For the degree of pleasure we can average the absolute value of $\mathrm{H}$. We find $\langle|\mathrm{H}|>=0.423$. This can be used to define the regimes of high pleasure, $\mathrm{H}><|\mathrm{H}|>$ and high displeasure, $\mathrm{H}<-<|\mathrm{H}|>$.

These figures show the raw data, which now are interpreted and analyzed.

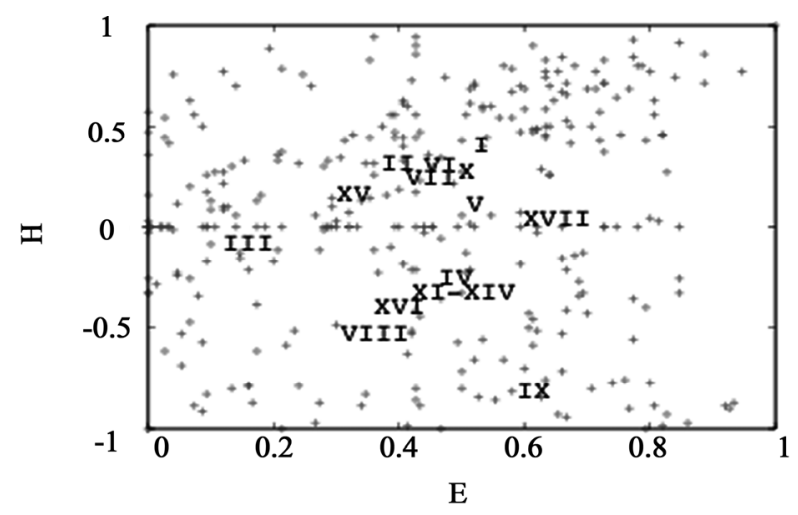

Figure 1.

Scatter plot of the arousal and pleasure degrees. For each one of the 34 subjects and for each question we represent the measured degrees of arousal and pleasure as the coordinates $(\mathrm{E}, \mathrm{H})$ of a point in a two-dimensional space. The Roman numerals indicate the location of the emotion classes defined according to Table 1).

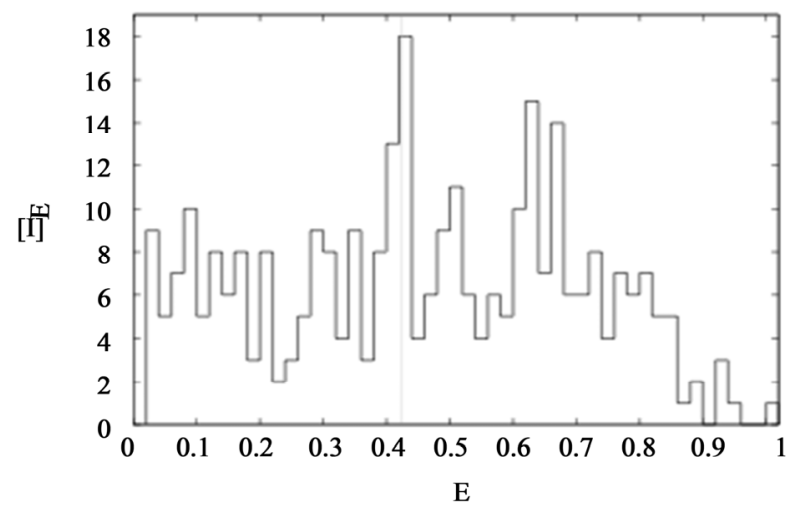

Figure 2.

Histogram of the distribution of values of the arousal measure E shown in the scatter plot of Figure 1 using a bin of size 0.02. The mean of this distribution is indicated by the vertical green line.

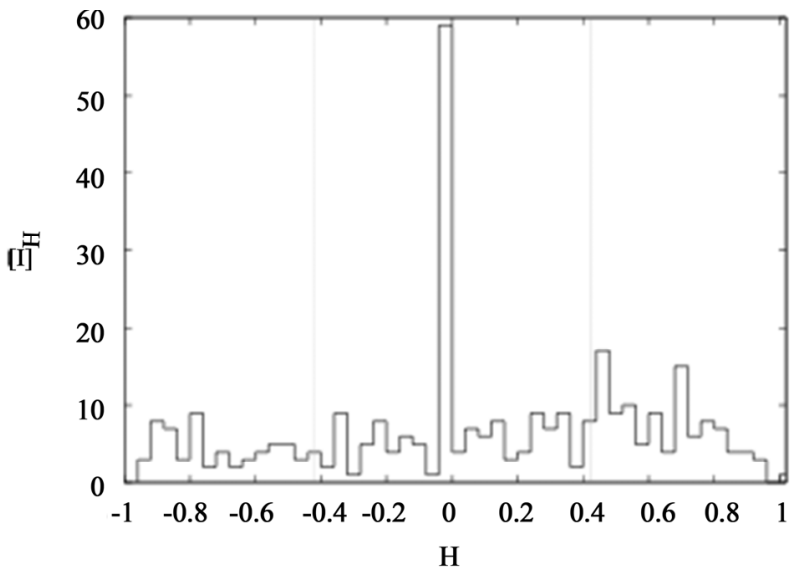

Figure 3.

Histogram of the distribution of values of the arousal measure $\mathrm{H}$ shown in the scatter plot of Figure 1 using a bin of size 0.02. The mean of this distribution is 0.058 . Here the symmetric vertical green lines indicate the mean and the negative mean of the absolute value of $\mathrm{H}$.

\section{The Arousal-Hedonicity Correlation}

Our previous analysis, indicate a strong correlation between the degrees of arousal (E) and pleasure $(\mathrm{H})$. Figure 4 presents these correlations for the 34 subjects that have completed the questionnaires (red crosses). We also show correlations obtained in the case $\mathrm{E}$ and $\mathrm{H}$ are chosen randomly and uniformly in the ranges $[0,1]$ and $[-1.1]$, respectively (green $\mathrm{x}$ ). The horizontal lines located at $\pm 1 / 3$ delimit region bounded by one standard deviation in the case $\mathrm{E}$ and $\mathrm{H}$ are independent random variables. We expect that on average about $32 \%$ of the correlation values would lay outside this region. For the random sample illustrated in the figure, only $21 \%$ of the values are outside those boundaries whereas $47 \%$ of the correlation values associated to the subjects' choices lay outside the one-standard deviation region. This indicates that the actual data are significantly more correlated than the random ones. We have studied the correlation between $\mathrm{E}$ and the absolute value of $\mathrm{H}$, i.e., $\operatorname{Cov}^{\alpha}(\mathrm{E}$, $|\mathrm{H}|)$, for each participant $\alpha$, and the results were qualitatively similar to those exhibited in Figure 4.

An interesting result revealed by Figure 4 is the enormous variation of the arousal-pleasure correlation among the subjects. For example, for the subject $\alpha=7$, arousal and pleasure are maximally correlated whereas for subject $\alpha=19$ these quantities as maximally uncorrelated. This result casts doubts on any conclusion based on the averaging over different subjects. Actually, if one averages the correlations over the 34 subjects we find $\sum \operatorname{Cor}^{\alpha}(\mathrm{E}, \mathrm{H}) / 34=0.132$ which is within the onestandard deviation zone of the random model.

A positive correlation indicates that the deviations of the pleasure and arousal degrees from the correspondent subject's means are in the same direction. It is interesting that the subject-dependent mean degrees of arousal and pleasure, $\langle\mathrm{E}\rangle_{\alpha}$ and $\langle\mathrm{H}\rangle_{\alpha}$, respectively, vary wildly among subjects, reflecting perhaps the personality and life-experience of them. These quantities are show in Figure 5.

\section{The Emotion Words}

As mentioned, 33 participants described the emotions they felt by a single emotion word. They used a total of 78 different 


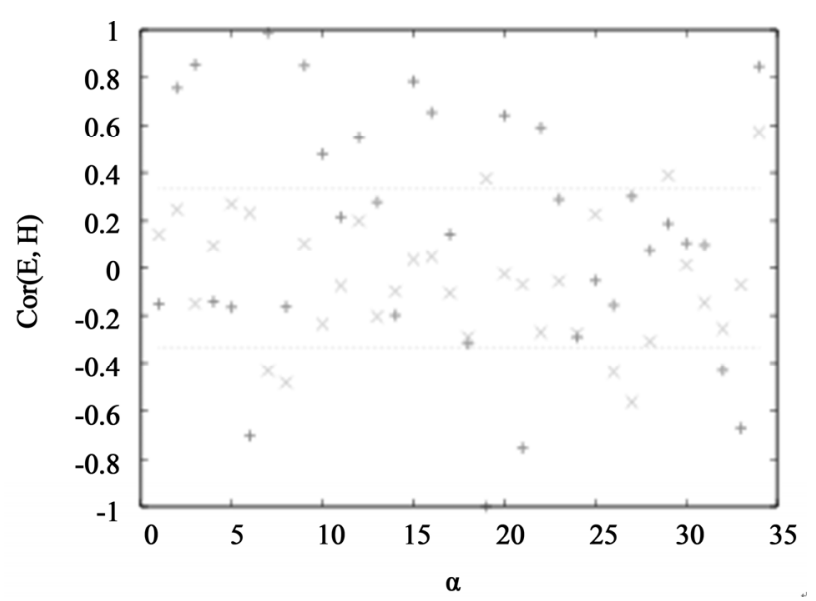

Figure 4.

Correlations between arousal and pleasure as defined in Equations (1) and (2) for each one of the 34 subjects (red crosses). The green $\mathrm{x} \mathrm{sym-}$ bols illustrate an outcome when the arousal and pleasure degrees are chosen randomly. The dashed horizontal lines, which are located at $\pm 1 / 3$, indicate one standard deviation of the correlation for the random case.

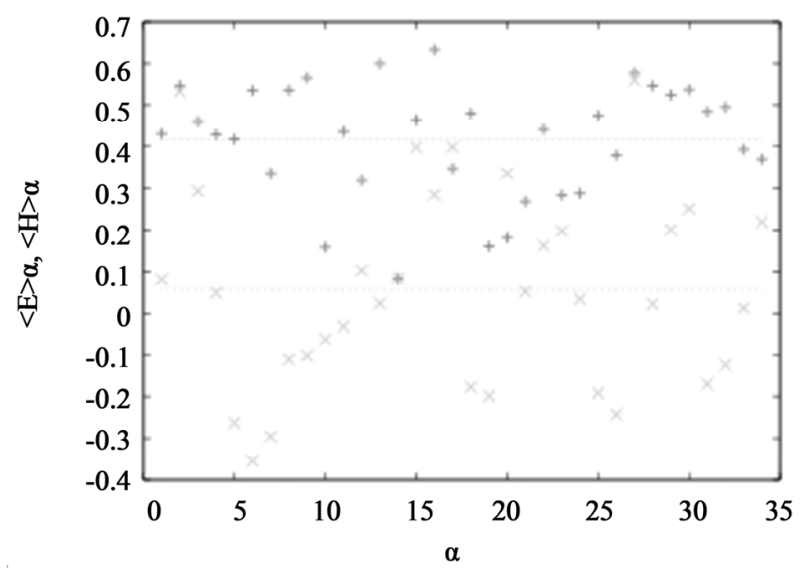

Figure 5.

Mean degree of arousal (red crosses) and mean degree of pleasure (green x's) for each subject. These means are calculated averaging over the measured values of E's and H's for the ten choice questions. The dashed horizontal lines indicate the average of these mean values over the 34 subjects.

emotion words for the 330 choice questions, which means that on the average each word was used 4 times. Considering that many of these words are synonymous or have very close meanings which could not be tell apart unless by an experienced psychologist, which was not the case of any of the participants, we decided to group the emotion words in 18 classes as described in Table 1. The numbers in the column Frequency indicate how many times a word in the corresponding class was used by the participants.

First, we address the issue of whether the subjects used these emotion words or, more correctly, classes, in a coherent way, in the sense that we expect that many participants use words within the same class to describe their emotions for a given choice question. To quantify this expectation, we calculate the probability that one selects two participants at random and they describe their emotions by words belonging to the same class
Table 1.

Basic emotion words and frequency of their use by participants to describe their $\mathrm{CD}$ emotions.

\begin{tabular}{|c|c|c|}
\hline Class & Words & Frequency \\
\hline I & $\begin{array}{l}\text { Joy, pleasure, satisfaction, delight, enthusiasm, } \\
\text { elation, excitement, luck, fun, well-being }\end{array}$ & 67 \\
\hline II & Desire, greed & 12 \\
\hline III & Indifference, minor interest, boredom & 57 \\
\hline IV & $\begin{array}{l}\text { Discomfort, embarrassment, uneasiness, distress, } \\
\text { impatience, guilt, anguish, displeasure, disarray }\end{array}$ & 33 \\
\hline $\mathrm{V}$ & Interest, curiosity, motivation, purpose, puzzling & 29 \\
\hline VI & Surprise & 9 \\
\hline VII & Hope, anticipation, expectation, waiting & 32 \\
\hline VIII & Rejection, repulsion & 3 \\
\hline IX & Furor, anger, wrath, irritation, indignation & 9 \\
\hline $\mathrm{X}$ & Serenity, relaxation, relief, safety, comfort & 11 \\
\hline $\mathrm{XI}$ & Sadness, nostalgia, fatalism, patience, weariness & 7 \\
\hline XII & $\begin{array}{l}\text { Concern, anxiety, fear, stress, nervousness, think- } \\
\text { ing, exasperated, doh! }\end{array}$ & 31 \\
\hline XIII & $\begin{array}{c}\text { Hesitation, incertitude, indecision, uncertainty, } \\
\text { unbelief, swindle }\end{array}$ & 10 \\
\hline XIV & $\begin{array}{l}\text { Disappointment, frustration, despair, disgust, dis- } \\
\text { couragement, perplexity }\end{array}$ & 9 \\
\hline $\mathrm{XV}$ & Challenge, difficulty & 2 \\
\hline XVI & Contempt, disdain & 6 \\
\hline XVII & Solidarity, commitment & 2 \\
\hline XVIII & Courage & 1 \\
\hline
\end{tabular}

for the same choice question $\mathrm{k}=1, \cdots, 10$. We recall that in this part of the experiment there are a total of 33 participants who can form 528 different pairs. So the desired probability is estimated by counting the number of pairs of subjects who choose words in the same class for each question $\mathrm{k}=1, \cdots, 10$ and dividing by 528. In Table 2 we show the results together with a realization of the random situation in which the subjects pick the classes I, $\cdots$, XVIII with probability proportional to the frequencies exhibited in Table 1.

Our goal has been to demonstrate that basic emotion words selected by participants do not adequately characterize their feelings of choice. If the probabilities in each column would be comparable this would be sufficient for our purpose. However, for 8 out of 10 cases real data result in a higher probability than the random model. Does it mean that basic emotions selected by subjects reflect the real difference in their feeling of choice? This psychological question, however cannot be answered from existing data. We have to acknowledge that if non-random probabilities from actual data are higher than random one, there could be emotional or other reasons affecting the choice nonrandomly. Therefore this test works only one way: if there is no statistically significant difference between the columns, that means that there is no emotional difference either. However, if 
the difference is statistically significant, we could not infer the psychological reason, and could only conclude that the test requires improvement.

The next step is to analyze statistical significance of the differences. We averaged columns 2 and 3 in Table 2 . The averaged probabilities for real data (column 2) and for the random model (column 3) are $P_{\mathrm{d}}=0.1670$ and $P_{\mathrm{r}}=0.1062$. The data probability is larger than the random one. Is this difference statistically significant? We approach this by generating $10^{5}$ random realizations similar to shown in the third column of Table 2 and computed their mean and standard deviation (of the probability distribution of $P_{\mathrm{r}}$ ). The results are $=0.1108$ and $\sigma_{\mathrm{r}}=0.004$. Hence $P_{\mathrm{d}}$ is 14 standard deviations away from $P_{\mathrm{r}}$, which means we can safely discard the possibility that the assignment of the emotion words to the choice questions were random. We would repeat again that psychological interpretations of these results so far remain uncertain.

As the next step we verify whether and how the emotionword classes presented in Table 1 are related to the degrees of arousal and pleasure. A simple way to approach this issue is to draw the histograms of the degrees of arousal and pleasure associated to a fixed emotion-word class. This is done in Figures 6-8 for emotion words in classes I, V and XII, respectively. (These figures can be compared with Figures $\mathbf{2}$ and 3, for which all classes are included.) The means and the standard deviations for each one of the 18 classes are presented in Table 3.

This table illustrates the inadequacy of characterizing emotions solely through the degrees of arousal and pleasure. In fact, in Figure 1 we show where the "centers of mass" of each class are located in the $(\mathrm{E}, \mathrm{H})$ space. We have omitted class XVIII because it overlaps with class VI. In addition, classes XI, XII, XIII and XIV are also too close to each other. The usefulness of such a representation is to get some insight about the similarity between emotions. Negative emotions represented by words in classes IV, VIII, XI-XIV, and XVI are close to each other. It is interesting that among these negative emotions contempt (XVI) is the closest to repulsion (VIII). It is also a welcome surprise that emotions such as curiosity (V), surprise (VI) and courage

Table 2.

Estimated probabilities of participants picking emotional word classes for each question, averaged over participants. Actual data are in column 2 and simulated random data are in column 3 .

\begin{tabular}{ccc}
\hline $\mathrm{k}$ & Data & Random \\
\hline 1 & 0.1648 & 0.0739 \\
2 & 0.2102 & 0.1382 \\
3 & 0.1534 & 0.0909 \\
4 & 0.2898 & 0.0682 \\
5 & 0.1326 & 0.1345 \\
6 & 0.1837 & 0.0966 \\
7 & 0.1061 & 0.1439 \\
8 & 0.1932 & 0.1136 \\
9 & 0.1439 & 0.0947 \\
10 & 0.0928 & 0.1079 \\
\hline
\end{tabular}

(XVIII) are also overlapping in the (E, H) space. There is definitely much to learn from such a representation but our statistics is still too poor to make any conclusive claim about the similarity between choice emotions. It is known from other studies specifically concentrated on basic emotions, that among approximately 150 emotional words there are between 5 and 30 different basic emotions (Ekman, 1999; Ortony \& Turner, 1990; Izard, 1992; Petrov et al., 2012).

\section{Conclusion}

Cognitive dissonance (CD) is a field of psychology intensely studied for more than 50 years. Nevertheless emotions of CD to our knowledge have not been studied. Experimental demonstration of $\mathrm{CD}$ emotions is possibly the main contribution of this paper. Choice decisions appear to be made in the hedonic dimension of consciousness (Cabanac et al., 1997, 2002, 2011); the hedonic experience takes place as an actual or an expected reward. In this paper we made a step toward exploring a new type of emotions, aesthetic emotions related to dissatisfaction of the knowledge instinct, or more specifically, emotions of CD related to contradictions between two pieces of knowledge. These emotions are different in principle from basic emotions. Whereas specific words exist to name basic emotions, there are no specific words for most emotions of cognitive dissonance. This creates difficulty to studying these emotions. This difficulty might be a reason that these emotions have not been systematically studied in psychological literature. Although the words "cognitive dissonance" have been used for long time, we emphasize again, emotions of cognitive dissonance have not been recognized as a special type of emotions different in principle from basic emotions.

Table 3.

Average and standard deviation of arousal and pleasure for each class.

\begin{tabular}{|c|c|c|}
\hline Class & Arousal & Pleasure \\
\hline $\mathrm{I}$ & $0.501 \pm 0.218$ & $0.417 \pm 0.324$ \\
\hline II & $0.400 \pm 0.210$ & $0.374 \pm 0.243$ \\
\hline III & $0.143 \pm 0.204$ & $-0.067 \pm 0.348$ \\
\hline IV & $0.472 \pm 0.225$ & $-0.213 \pm 0.543$ \\
\hline V & $0.530 \pm 0.190$ & $0.151 \pm 0.455$ \\
\hline VI & $0.439 \pm 0.286$ & $0.308 \pm 0.348$ \\
\hline VII & $0.410 \pm 0.273$ & $0.274 \pm 0.402$ \\
\hline VIII & $0.307 \pm 0.327$ & $-0.514 \pm 0.364$ \\
\hline IX & $0.550 \pm 0.250$ & $-0.630 \pm 0.505$ \\
\hline $\mathrm{X}$ & $0.481 \pm 0.248$ & $0.274 \pm 0.505$ \\
\hline XI & $0.512 \pm 0.315$ & $-0.308 \pm 0.683$ \\
\hline XII & $0.456 \pm 0.241$ & $-0.241 \pm 0.547$ \\
\hline XIII & $0.455 \pm 0.220$ & $-0.317 \pm 0.407$ \\
\hline XIV & $0.522 \pm 0.186$ & $-0.348 \pm 0.578$ \\
\hline XV & $0.300 \pm 0.233$ & $0.171 \pm 0.428$ \\
\hline XVI & $0.400 \pm 0.262$ & $-0.209 \pm 0.544$ \\
\hline XVII & $0.597 \pm 0.097$ & $0.050 \pm 0.378$ \\
\hline XVIII & $0.406 \pm 0.000$ & $0.328 \pm 0.000$ \\
\hline
\end{tabular}




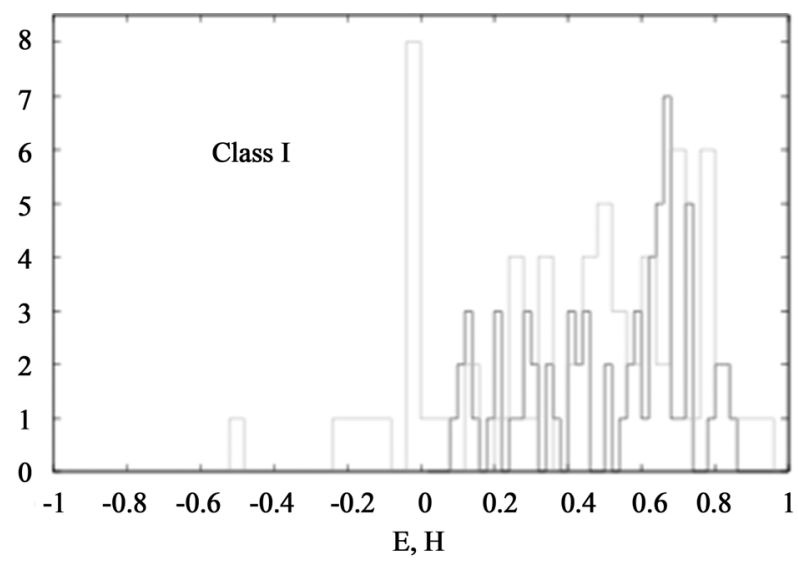

Figure 6.

Histograms of the degrees of arousal (red lines) and pleasure (green lines) restricted to choices associated to emotion words belonging to class I, e.g., joy, pleasure, enthusiasm, etc. For this class, the mean degree of arousal is 0.501 and the mean degree of pleasure is 0.417 .

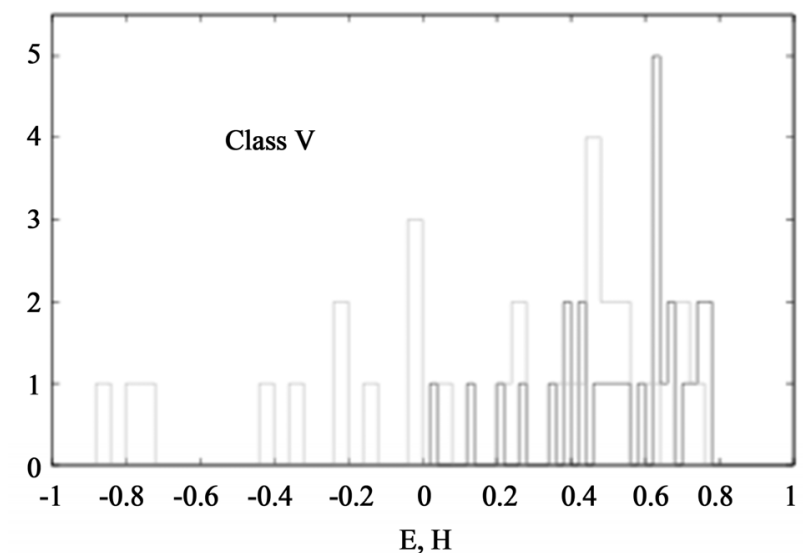

Figure 7.

Histograms of the degrees of arousal (red lines) and pleasure (green lines) restricted to choices associated to emotion words belonging to class V, e.g., curiosity, interest, purpose etc. For this class, the mean degree of arousal is 0.530 and the mean degree of pleasure is 0.151 .

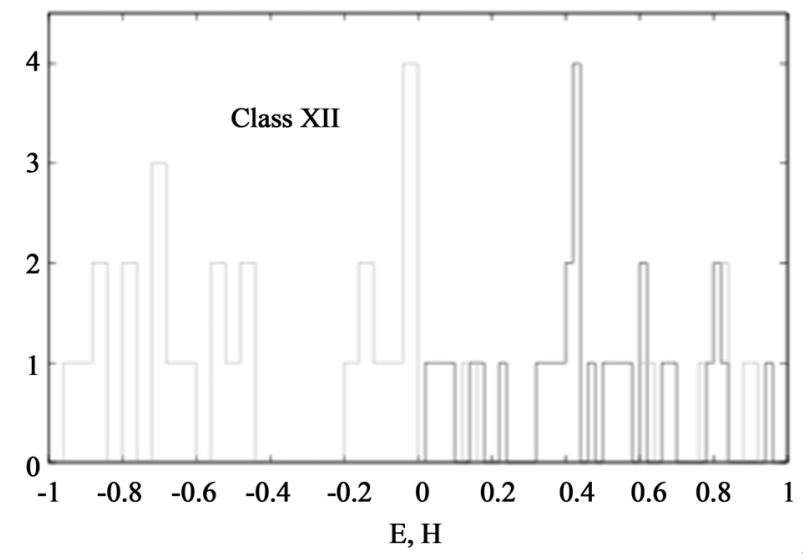

Figure 8.

Histograms of the degrees of arousal (red lines) and pleasure (green lines) restricted to choices associated to emotion words belonging to class XII, e.g., fear, anxiety, concern etc. For this class, the mean degree of arousal is 0.456 and the mean degree of pleasure is -0.241 .
In this paper, following (Grossberg \& Levine, 1987), we consider emotions as feelings and mental states related to neural signals, which indicate to various brain regions satisfaction or dissatisfaction of fundamental organism needs. Mechanisms measuring these needs we call instincts. Basic emotions are mostly related to bodily needs, whereas aesthetic emotions are related to need for knowledge or knowledge instinct (KI). This is a fundamental theoretical difference between basic and aesthetic emotions. Also in (Perlovsky, 2006c, 2010a, 2012c, 2012d) the arguments were presented that emotions of cognitive dissonance could be in some way similar to musical emotions. Steps to demonstrating this experimentally have been made in (Masataka et al., 2012a, 2012b; Perlovsky, Cabanac et al., 2012). It has been demonstrated that music helps resolve $\mathrm{CD}$ emotions of discomfort, so that contradictions in knowledge can be tolerated (Masataka et al., 2012a, 2012b; Perlovsky, Cabanac et al., 2012). This made possible accumulation of knowledge and evolution of cultures. The "Mozart effect" has been demonstrated to be caused by resolving CD. Still many questions remain challenges for future research.

The main experiential argument separating basic and aesthetic emotions of cognitive dissonances in this paper are examples of the following type of choices. A choice between different types of work could be difficult and experienced as negative $C D$ emotions, even if each alternative separately is experienced as positive basic emotions. In this paper our results are inconclusive with regard to qualitative difference between basic and aesthetic emotions. It is possible that standard methods of exploring emotions, arousal and pleasure plots, characterizing each aesthetic emotion of cognitive dissonance by a single basic emotion - these methods may not be sensitive enough for fine distinctions required to differentiate details of aesthetic emotions. One has to keep in mind that mechanisms of basic emotions are hundreds of millions or even billions years old, whereas $\mathrm{CD}$ emotions are due to recently evolved mechanisms, probably less than millions years old.

The main experimental result of this paper is "instrumentalizing" aesthetic emotions of cognitive dissonance, by presenting subject questions as mental choices between two alternatives. In future we suggest more refined measures, such as 1) using more refined splitting of basic emotions in classes than the relatively subjective groupings in Table $2 ; 2$ ) characterizing each emotion of choice by several basic emotions; 3 ) measuring distances-similarities between various choice emotions and then use these distances for analyzing properties of the aesthetic emotional space.

\section{REFERENCES}

Balaskó, M., \& Cabanac, M. (1998). Grammatical choice and affective experience in a second-language test. Neuropsychobiology, 37, 205210. doi:10.1159/000026504

Bechara, A., Damasio, A. R., Damasio, H., \& Anderson, S. W. (1994). Insensitivity to future consequences following dam-age to human prefrontal cortex. Cognition, 50, 7-15. doi:10.1016/0010-0277(94)90018-3

Bonniot-Cabanac, M.-C., \& Cabanac, M. (2009). Pleasure in decision making situations: Politics and gambling. Journal of Risk Research, 12, 619-645. doi:10.1080/13669870802579798

Bonniot-Cabanac, M.-C., \& Cabanac, M. (2010). Do government officials decide more rationally than the rest of us? A study using participants from the legislature, the executive, and the judiciary. Social Behavior \& Personality, 38, 1147-1152. 
doi:10.2224/sbp.2010.38.8.1147

Bonniot-Cabanac, M.-C., Cabanac, M., Fontanari, J. F., \& Perlovsky, L.I. (2012). A structural model of emotions of cognitive dissonances. Neural Networks, 32, 57-64.

Cabanac, M. (2002). What is emotion? Behavioural Processes, 60, 69-83.

Cabanac, M., \& Bonniot-Cabanac, M. C. (2007). Decision making: Rational or hedonic? Behavioral and Brain Functions, 3, 1-45. doi:10.1186/1744-9081-3-45

Cabanac, M., Cabanac, A. J., \& André Parent, A. (2009). When in phylogeny did consciousness emerge? Behavioral and Brain Research, 198, 267-272. doi:10.1016/j.bbr.2008.11.028

Cabanac, M., \& Bonniot-Cabanac, M.-C. (2011). Hedonicity and memory of odors. International Journal of Psychological Studies, 3, 178-185.

Cabanac, M., Guillaume, J., Balaskó, M., \& Fleury, A. (2002). Pleasure in decision making situations. Biomed Central, $2,7$.

Cabanac, M., Pouliot, C., \& J. Everett. (1997). Pleasure as a sign of efficacy of mental activity. European Psychologist, 2, 226-234. doi:10.1027/1016-9040.2.3.226

Damasio, H., Grabowski, T., Frank, R., Galaburda, A. M., \& Damasio, A. R. (1994). The return of phineas gage: Clues about the brain from the skull of a famous patient. Science, 264, 1102-1105. doi:10.1126/science. 8178168

Ekman, P. (1957). A methodological discussion of nonverbal behavior. Journal of Psychology, 43, 141-149. doi: $10.1080 / 00223980.1957 .9713059$

Ekman, P. (1999). Basic emotions. In T. Dalgleish and M. Power (Eds.), Handbook of Cognition and Emotion. Sussex: John Wiley \& Sons.

Festinger, L. (1957). A theory of cognitive dissonance. Stanford, CA: Stanford University Press.

Gratch, J., Marsella, S., \& Petta, P. (2009). Modeling the cognitive antecedents and consequences of emotion. Cognitive Systems Research, 10, 1-5. doi:10.1016/j.cogsys.2008.06.001

Grossberg, S., \& Levine, D. (1987). Neural dynamics of attentionally modulated Pavlovian conditioning: Blocking, interstimulus interval, and secondary reinforcement. Applied Optics, 26, 5015-5030. doi:10.1364/AO.26.005015

Kant, I. (1790). The critique of judgment. Amherst, MA: Prometheus Books.

Izard, C. E. (1992). Basic emotions, relations among emotions, and emotion-cognition relations. Psychological Review, 99, 561-565. doi:10.1037/0033-295X.99.3.561

Johnson-Laird, P. N., \& Oatley, K. (1992). Basic emotions: a cognitive science approach to function, folk theory and empirical study. Cognition and Emotion, 6, 201-223. doi: $10.1080 / 02699939208411069$

Juslin, P. N., \& Västfjäll, D. (2008) Emotional responses to music: The need to consider underlying mechanisms. Behavioral and Brain Sciences, 31, 559-575.

Kovalerchuk, B., Perlovsky, L., \& Wheeler, G. (2012). Modeling of phenomena and dynamic logic of phenomena. Journal of Applied Non-classical Logics, 22, 51-82. doi:10.1080/11663081.2012.682439

Lazarus, R. S. (1991). Emotion and adaptation. New York: Oxford University Press.

Levine, D. S., \& Perlovsky, L. I. (2008). Neuroscientific insights on biblical myths: Simplifying Heuristics versus Careful Thinking: Scientific analysis of millennial spiritual issues. Zygon, Journal of Science and Religion, 43, 797-821.

Levine, D. S., \& Perlovsky, L. I. (2010). Emotion in the pursuit of understanding. International Journal of Synthetic Emotions, 1, 1-11. doi:10.4018/jse.2010070101

Masataka, N., \& Perlovsky, L. I. (2012a). Music can reduce cognitive dissonance. Nature Precedings.

Masataka, N., \& Perlovsky, L. I. (2012b). The efficacy of musical emotions provoked by Mozart's music for the reconciliation of cognitive dissonance. Scientific Reports (in press). doi: $10.1038 /$ srep00694

Ortony, A., Clore, G. L., \& Foss, M. A. (1987). The referential structure of the affective lexicon. Cognitive Science, 11, 341-364. doi: $10.1207 / \mathrm{s} 15516709 \operatorname{cog} 1103 \_4$
Ortony, A., \& Turner, T. J. (1990). What's basic about basic emotions? Psychological Review, 97, 315-331. doi:10.1037/0033-295X.97.3.315

Perlovsky, L. I. (1997). Physical concepts of intellect. Proceedings of Russian Academy of Sciences, 354, 320-323.

Perlovsky, L. I. (2000). Beauty and mathematical intellect. Zvezda, 9, 190-201.Perlovsky, L. I. (2001). Mystery of sublime and mathematics of intelli- gence. Zvezda, 8, 174-190.

Perlovsky, L. I. (2002a). Physical theory of information processing in the mind: Concepts and emotions. SEED on Line Journal, 2 36-54Perlovsky, L. I. (2002b). Aesthetics and mathematical theories of intellect. Iskusstvoznanie, 2, 558-594.Perlovsky, L. I. (2004). Integrating language and cognition. IEEE Connections, Feature Article, 2, 8-12.

Perlovsky, L. I. (2006a). Toward physics of the mind: Concepts, emotions, consciousness, and symbols. Physics of Life Reviews, 3, 22-55. doi:10.1016/j.plrev.2005.11.003

Perlovsky, L. I. (2006b). Fuzzy dynamic logic. New Mathematics and Natural Computation, 2, 43-55. doi:10.1142/S1793005706000300

Perlovsky, L. I. (2006c). Music-The first priciple. URL (last checked 3 December 2011). http://www.ceo.spb.ru/libretto/kon lan/ogl.shtml

Perlovsky, L. I. (2007a). Cognitive high level information fusion. Information Sciences, 177, 2099-2118. doi:10.1016/j.ins.2006.12.026

Perlovsky, L. I. (2007b). Evolution of Languages, Consciousness, and Cultures. IEEE Computational Intelligence Magazine, 2, 25-39. doi:10.1109/MCI.2007.385364

Perlovsky, L. I. (2007c). The Mind vs. logic: Aristotle and zadeh. Society for Mathematics of Uncertainty, Critical Review, 1, 30-33.

Perlovsky, L. I. (2008). Music and consciousness. Journal of Arts, Sciences and Technology, 41, 420-421.

Perlovsky, L. I. (2009a). Language and cognition. Neural Networks, 22, 247-257. doi:10.1016/j.neunet.2009.03.007

Perlovsky, L. I. (2009b). Language and emotions: Emotional SapirWhorf hypothesis. Neural Networks, 22, 518-526. doi:10.1016/j.neunet.2009.06.034

Perlovsky, L. I. (2009c). "Vague-to-crisp" neural mechanism of perception. IEEE Transactions of Neural Networks, 20, 1363-1367. doi:10.1109/TNN.2009.2025501

Perlovsky, L. I. (2010a). Musical emotions: Functions, origin, evolution. Physics of Life Reviews, 7, 2-27. doi:10.1016/j.plrev.2009.11.001

Perlovsky, L. I. (2010b). Neural mechanisms of the mind, aristotle, zadeh, \& fMRI. IEEE Transactions of Neural Networks, 21, 718-733. doi:10.1109/TNN.2010.2041250

Perlovsky, L. I. (2010c). Intersections of mathematical, cognitive, and aesthetic theories of mind. Psychology of Aesthetics, Creativity, and the Arts, 4, 11-17. doi:10.1037/a0018147

Perlovsky, L. I. (2010d). The Mind is not a kludge. Skeptic, 15, 51-55

Perlovsky, L. I. (2010e). Joint acquisition of language and cognition; WebmedCentralBRAIN, 1, WMC00994. http://www.webmedcentral.com/article_view/994

Perlovsky L. I. (2010f). Physics of the mind: Concepts, emotions, language, cognition, consciousness, beauty, music, and symbolic culture. WebmedCentral Psychology 2010, 1, WMC001374.

Perlovsky, L. I. (2010g). Beauty and art, Cognitive function, evolution, and mathematical models of the mind. WebmedCentral Psychology 2010, 1, WMC001322.

Perlovsky, L. I. (2011a). Music, cognitive function, origin, and evolution of musical emotions. WebmedCentral Psychology 2011, 2, WMC001494.

Perlovsky, L. I. (2011b). Consciousness and free will: A scientific possibility due to advances in cognitive science. WebmedCentral Psychology 2011, 2, WMC001539

Perlovsky, L. I. (2011c). Language, emotions, and cultures: Emotional Sapir-Whorf hypothesis. WebmedCentral Psychology 2011, 2, WMC001580.

Perlovsky, L. I. (2011d). Computational intelligence applications for defense. IEEE Computational Intelligence Magazine, 6, 20-28. doi:10.1109/MCI.2010.939581

Perlovsky L. I. (2011e). Language and cognition interaction neural mechanisms. Computational Intelligence and Neuroscience, 3, 454587. doi:10.1155/2011/454587 
Perlovsky, L. I. (2011f). High cognitive emotions in language prosody. Physics of Life Reviews, 8, 408-409. doi:10.1016/j.plrev.2011.10.007

Perlovsky, L. I. (2011g). Abstract concepts in language and cognition, Physics of Life Reviews, 8, 375-376. doi:10.1016/j.plrev.2011.10.006

Perlovsky, L. I. (2012a). Free will and advances in cognitive science. Open Journal of Philosophy (OJPP), 2, 32-37. doi:10.4236/ojpp.2012.21005

Perlovsky, L. I. (2012b). The cognitive function of emotions of spiritually sublime. Review of Psychology Frontier, 1, 1-10.

Perlovsky L. I. (2012c). Emotions of "higher" cognition. Comment to lindquist at the brain basis of emotion: A meta-analytic review. Brain and Behavior Sciences, 35, 157-158. doi:10.1017/S0140525X11001555

Perlovsky, L. I. (2012d). Cognitive function, origin, and evolution of musical emotions. Musicae Scientiae, 16, 185-199. doi: $10.1177 / 1029864912448327$

Perlovsky, L. I. (2012e). Cognitive function of music: Part I. Interdisciplinary Science Reviews, 37, 129-142. doi: $10.1179 / 0308018812 Z .00000000010$

Perlovsky, L. I. (2012f). Nonlinear dynamics and higher cognitive mental functions. Physics of Life Reviews, 9, 74-75. doi:10.1016/j.plrev.2011.12.004

Perlovsky, L. I. (2012g). Cognitive function of musical emotions. Psychomusicology (in press).

Perlovsky, L. I. (2012h). Mirror neurons, language, and embodied cognition. Neural Networks (in press).

Perlovsky, L. I. (2012i). Brain: Conscious and unconscious mechanisms of cognition, emotions, and language. Brain Sciences (in press).

Perlovsky, L. I. (2012j). The cognitive function of emotions of spiritually sublime. Review of Psychology Frontier, 1, 1-10.

www.j-psy.org.

Perlovsky, L. I. (2012k). Emotionality of languages affects evolution of cultures. Review of Psychology Frontier (in press).

Perlovsky, L. I., Bonniot-Cabanac, M., \& Cabanac, M. (2010). Curiosity and pleasure. WebmedCentral Psychology 2010, 1, WMC001275

Perlovsky, L. I., Cabanac, A., Bonniot-Cabanac, M.-C., \& Cabanac, M. (2012). Mozart effect, cognitive dissonance, and origin of music. Behavioral Brain Research (in press).

Perlovsky, L. I., \& Ilin, R. (2010). Neurally and mathematically motivated architecture for language and thought. The Open Neuroimaging Journal, 4, 70-80. doi:10.2174/1874440001004020070

Perlovsky, L. I., \& Ilin, R. (2012a). Mathematical model of grounded symbols: Perceptual symbol system. Journal of Behavioral and Brain Science, 2, 195-220. doi:10.4236/jbbs.2012.22024

Perlovsky, L. I., \& Ilin, R. (2012b). CWW, language, and thinking. New Mathematics and Natural Computations (in press).

Perlovsky, L. I., \& Levine, D. (2012). The drive for creativity and the escape from creativity: Neurocognitive mechanisms. Cognitive Computation, 4, 292-305. doi:10.1007/s12559-012-9154-3

Petrov, S., Fontanari, J. F., \& Perlovsky, L. I. (2012). Subjective emotions vs. verbalizable emotions in web texts. International Journal of Psychology and Behavioral Sciences, 2, 173-184.

Plutchik, R. (1962). The emotions: Facts, theories and a new model. New York: Random House.

Plutchik, R. (1980). A general psychoevolutionary theory of emotion. In R. Plutchik, \& H. Kellerman (Eds.), Emotion: Theory, Research, and Experience (pp. 3-33). New York: Academic Press.

Ramírez, J. M., Bonniot-Cabanac, M.-C., \& Cabanac, M. (2005) Can aggression provide pleasure? European Psychologist, 10, 136-145.

Ramírez, J. M., Millana, L., Toldos-Romero, M. P., Bonniot-Cabanac, M.-C., \& Cabanac, M. (2009) The pleasure of being aggressive in incarcerated criminals. The Open Criminology Journal, 2, 1-9.

Reisenzein, R. (2009). Emotions as metarepresentational states of mind: Naturalizing the belief-desire theory of emotion. Cognitive Systems Research, 10, 6-20. doi:10.1016/j.cogsys.2008.03.001

Russel, J. A. (1980). A circumplex model of affect. Journal of Personality and Social Psychology, 39, 1161-1178.

Russel, J. A. (1989). Measures of emotion. In R. Plutchik, H. Kelerman (Eds.), Emotion: The measurement of Emotion (pp. 83-110). New York: Academic Press. doi: 10.1037/h0077714

Scherer, K. R., Schorr, A., \& Johnstone, T. (2001). Appraisal processes in emotion: Theory, methods, research. New York: Oxford University Press.

Tooby, J., \& Cosmides, L. (1990). The past explains the present: Emotional adaptations and the structure of ancestral environment. Ethology and Sociobiology, 11, 375-424. doi:10.1016/0162-3095(90)90017-Z

Tikhanoff, V., Fontanari, J. F., Cangelosi, A., \& Perlovsky, L. I. (2006) Language and cognition integration through modeling field theory: Category formation for symbol grounding. In S. Kollias, A. Stafylopatis, W. Duch, \& E. Oja (Eds.), Book Series in Computer Science. Heidelberg: Springer.

Vityaev, E. E., Perlovsky, L. I., Kovalerchuk, B. Y., \& Speransky, S. O. (2011). Probabilistic dynamic logic of the mind and cognition. Neuroinformatics, 5, 1-20. 


\section{Appendix}

The 10 items of Questionnaire E aiming at measuring the degree of arousal of the evoked emotion are presented below:

1) Focus on what you feel when you are asked to make a choice: with the duck with orange what do you prefer of red wine or white wine? Do you feel an emotion at the idea of this choice? Indicate its intensity on the line below.

0 Max

2) Focus on what you feel when you are asked to make a choice: what do you prefer the cinema or theater? Do you feel an emotion at the idea of this choice? Indicate its intensity on the line below.

0 Max

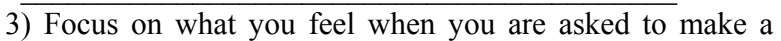
choice: what do you prefer of the holiday sea or in the mountain? Do you feel an emotion at the idea of this choice? Indicate its intensity on the line below.

0

Max

4) Focus on what you feel when you are asked to make a choice: what do you prefer of the holiday sea or in the mountain? Do you feel an emotion at the idea of this choice? Indicate its intensity on the line below.

0 Max

5) Focus on what you feel when you are asked to make a choice of career: what do you prefer: a relatively poorly paid work but secure or a very well-paid work but at risk of loss of employment. Do you feel an emotion at the idea of this choice? Indicate its intensity on the line below.

0 Max

6) Focus on what you feel when you are asked to make a choice: what do you prefer to hear a Sonata for violin or piano? Do you feel an emotion at the idea of this choice? Indicate its intensity on the line below.

0
7) Focus on what you feel when you are asked to make a choice: your work requires you to learn a Scandinavian language; what do you prefer the Norwegian or Swedish? Do you feel an emotion at the idea of this choice? Indicate its intensity on the line below.

0 Max

8) Focus on what you feel when you are asked to make a choice: to treat an illness with what do you prefer the quick surgery or medication extended? Do you feel an emotion at the idea of this choice? Indicate its intensity on the line below.

0 Max

9) Focus on what you feel when you are asked to make a choice: do you prefer very expensive insurance but comprehensive or cheaper but with gaps? Do you feel an emotion at the idea of this choice? Indicate its intensity on the line below.

0 Max

10) Focus on what you feel when you are asked to make a choice about the elections: the Right party, which guarantees the security or the Left party, which promotes a sharing social? Do you feel an emotion at the idea of this choice? Indicate its intensity on the line below.

0 Max

In addition, in order to measure the degree of pleasure in making that choice, the same set of choice-questions was presented to the participants except that they were asked to indicate in the following straight line, after reading each item,

$$
\text { - } 0 \text { ___ }+
$$

the degree of pleasure. Here the - sign indicates a most unpleasant choice and the + sign a most pleasant one, and the distance from zero was the analog magnitude rating of hedonicity. This is an example of item from Questionnaire $H$ that contained the same 10 items as Questionnaire E. 